\title{
Two-dimensional Bloch electrons in perpendicular magnetic fields: An exact calculation of the Hofstadter butterfly spectrum
}

\author{
S. Janecek,,$^{1,2,3,4, *}$ M. Aichinger, ${ }^{3,5}$ and E. R. Hernández ${ }^{1,2}$ \\ ${ }^{1}$ Instituto de Ciencia de Materiales de Madrid (ICMM-CSIC), Campus de Cantoblanco, 28049 Madrid, Spain \\ ${ }^{2}$ Institut de Ciencia de Materials de Barcelona (ICMAB-CSIC), Campus de Bellaterra, 08193 Barcelona, Spain \\ ${ }^{3}$ Johann Radon Institute for Computational and Applied Mathematics (RICAM), Austrian Academy of Sciences, \\ Altenberger Strasse 69, A-4040 Linz, Austria \\ ${ }^{4}$ MathConsult GmbH, Altenberger Strasse 69, A-4040 Linz, Austria \\ ${ }^{5}$ Uni Software Plus GmbH, Kreuzstrasse 15a, A-4040 Linz, Austria
}

(Received 9 March 2013; published 25 June 2013)

\begin{abstract}
The problem of two-dimensional, independent electrons subject to a periodic potential and a uniform perpendicular magnetic field unveils surprisingly rich physics, as epitomized by the fractal energy spectrum known as Hofstadter's butterfly. It has hitherto been addressed using various approximations rooted in either the strong potential or the strong field limiting cases. Here, we report calculations of the full spectrum of the single-particle Schrödinger equation without further approximations. Our method is exact, up to numerical precision, for any combination of potential and uniform field strength. We first study a situation that corresponds to the strong potential limit, and compare the exact results to the predictions of a Hofstadter-like model. We then go on to analyze the evolution of the fractal spectrum from a Landau-like nearly free electron system to the Hofstadter tight-binding limit by tuning the amplitude of the modulation potential.
\end{abstract}

DOI: 10.1103/PhysRevB.87.235429

PACS number(s): 73.50.Jt, 73.43.Lp, 75.47.-m

\section{INTRODUCTION}

The motion of electrons in a crystalline solid subject to an external magnetic field has been considered since the early days of quantum mechanics. ${ }^{1}$ The field splits the crystal's electronic bands into subbands and internal minigaps; when plotted as a function of the field, the energetic arrangement of these subbands forms a fractal structure that has come to be known as Hofstadter's butterfly. ${ }^{2}$ Its underlying principle is the frustration of two competing symmetries, namely, the periodicity of the lattice and the symmetry of the Landau orbits, which are characterized by their associated length scales, the lattice constant $a$ and the magnetic length $l_{\mathrm{B}}=\sqrt{\hbar /(e B)}$. To reach the interesting regime $l_{\mathrm{B}} \approx a$ at laboratory fields, experimentalists have resorted to superlattices patterned on top of two-dimensional electron gases (2DEGs), ${ }^{3,4}$ as originally suggested by Hofstadter. ${ }^{2}$ Such experiments are quite formidable tasks due to conflicting requirements on the sample. ${ }^{3}$ It was recently suggested ${ }^{5}$ that moiré patterns in a twisted bilayer graphene could be tailored to obtain superlattices with periodicities in the range of a few tens of nanometers, with which magnetic fields of only a few tesla would be required to observe the butterfly spectrum. Very recently, this suggestion has lead to the experimental realization of devices based on hexagonal$\mathrm{BN}$ and graphene bilayer moiré superlattices that enable unprecedented experimental access to the fractal spectrum. ${ }^{6,7}$

Patterns similar to the Hofstadter butterfly have been observed or predicted to occur in a variety of very different systems with frustrated symmetries, such as microwaves transmitted through a waveguide with a periodic arrangement of scatterers, ${ }^{8}$ the electronic ${ }^{9}$ and vibrational ${ }^{10}$ spectra of incommensurate crystals, ultracold atoms in optical lattices, ${ }^{11}$ and photonic crystals. ${ }^{12,13}$ The topological protection of the quantum Hall phase in a photonic crystal has been shown to improve the performance of optical delay lines and to overcome limitations related to disorder in photonic technologies. ${ }^{13}$
From a theoretical perspective, this problem has been chiefly approached by approximations starting from two complementary limits, considering either the influence of a weak magnetic field on the band structure resulting from a strongly varying lattice potential, ${ }^{14,15}$ or the influence of a small modulation potential on the Landau-quantized electrons in a strong field. ${ }^{15,16}$ In the strong potential limit, one typically starts with a tight-binding (TB) approximation for a single band of the zero-field problem, $E(\mathbf{k})$, where $\mathbf{k}$ is the crystal momentum. Then, an effective Hamiltonian for the magnetic field problem is generated from $E(\mathbf{k})$ through the Peierls substitution $\mathbf{k} \rightarrow(\mathbf{p}+e \mathbf{A}) / \hbar,{ }^{1,17}$ where $(\mathbf{p}+e \mathbf{A})$ is the dynamical momentum operator in a magnetic field, $\mathbf{p}$ the canonical momentum operator, and $\mathbf{A}$ the vector potential. This procedure was used, among others, by Hofstadter in his seminal article for a nearest-neighbor (NN) TB model of the $2 \mathrm{D}$ square lattice. ${ }^{2}$ There are a number of simplifications inherent to this approach: (i) the $\mathrm{TB}$ approximation of the zero-field band structure, (ii) the restriction to electrons in a single band, and (iii) the neglect of the diamagnetic energy of the TB orbitals and the field dependence of the TB hopping integrals. This has been shown to lead to quantitative as well as qualitative errors for both nearly free and tightly bound two-dimensional electrons. ${ }^{18-21}$ Generalization of the effective Hamiltonian approach has turned out to be difficult, see, e.g., Ref. 22. Surprisingly, the strong field approach is closely related to the strong potential one: if potential-induced coupling between different Landau levels is neglected, the same secular equation is obtained, but with the magnetic field replaced by its inverse. ${ }^{15}$ Including such coupling has a profound effect on the calculated energy spectrum, ${ }^{23,24}$ even for weak coupling strength. The resulting rearrangement of the Hofstadter butterfly has recently been confirmed by experiments. ${ }^{4}$ We take these results as a strong indication that in order to understand the experimental data, $3,4,6,7$ it is necessary to go beyond the approximations described above. 
In this article, we describe a computational methodology that allows to obtain the single-electron spectrum of this system for any combination of periodic potential and uniform magnetic field strength, without relying on any of the approximations incurred in previous methodologies. Our results are thus exact, up to numerical precision. We use this methodology to study first a situation that corresponds to the strong potential limit, and compare our results to the predictions obtained with the Hofstadter approach. We then go on to analyze the evolution of the fractal energy spectrum as the periodic potential is changed from a nearly flat case, corresponding to the Landau-like nearly free electron situation, to the Hofstadter TB limit of a strongly corrugated potential. The intermediate stages of this evolution are not accessible to the above approximations.

The structure of this paper is as follows: in Sec. II, we outline the necessary theoretical background; we discuss how in the case of rational field values a generalized Bloch condition holds on a suitably chosen magnetic cell. Some of the necessary steps in the derivation are spelled out in more detail in an appendix. Section III discusses how this generalized Bloch condition can be exploited to construct a practical numerical scheme, and details of our particular implementation are provided; brief descriptions of the calculation of the Hall conductance and of the Hofstadter low-field approximation are also given. Our main results are presented in Sec. IV, where we first apply our method to a situation that is amenable to treatment with the Hofstadter strong potential/weak field approach, and compare the results of both methods. Secondly, we present results for a series of different potential strengths, ranging from the weakly modulated potential, closely corresponding to the Landau limit of free electrons in a magnetic field, to the strongly corrugated potential limit, and discuss the changing nature of the spectrum as the potential is varied. Finally, our conclusions are presented in Sec. V.

\section{THEORETICAL BACKGROUND}

The motion of independent electrons is described by the single-particle Schrödinger equation,

$$
H \psi(\mathbf{r}) \equiv\left[\frac{1}{2 m} \Pi^{2}+V(\mathbf{r})\right] \psi(\mathbf{r})=E \psi(\mathbf{r}),
$$

where $H$ is the Hamiltonian, $\psi(\mathbf{r})$ is an eigenstate with energy $E, \boldsymbol{\Pi}=\mathbf{p}+e \mathbf{A}(\mathbf{r})$ is the dynamical momentum operator and $\mathbf{A}(\mathbf{r})$ is the vector potential corresponding to the magnetic field, $\mathbf{B}=\nabla \times \mathbf{A}$. We take the field to be uniform and oriented along the $z$ direction, $\mathbf{B}=B \mathbf{e}_{z}$. The electrons are restricted to the two-dimensional (2D) $x y$ plane, and the external potential $V(\mathbf{r})$ is periodic on a Bravais lattice defined by vectors

$$
\mathbf{R}_{\mathbf{n}}=j \mathbf{a}+k \mathbf{b}, \quad \mathbf{n}=(j, k) \in \mathbb{Z}^{2},
$$

where $\mathbb{Z}$ represents the Zahl set of positive and negative integers. In the absence of an external magnetic field, Bloch's theorem, which results from the commutation of the lattice translations with the Hamiltonian, allows to restrict the calculation of the eigenfunctions $\psi(\mathbf{r})$ to one primitive cell of the lattice in Eq. (2). As these translations do not leave the vector potential $\mathbf{A}(\mathbf{r})$ invariant, they no longer commute with $H$ when a magnetic field is present; consequently, the eigenfunctions $\psi(\mathbf{r})$ are not Bloch waves. By combining a lattice translation with a suitable gauge transformation to counteract its effect upon the vector potential, one can define magnetic translation operators, $T_{A}\left(\mathbf{R}_{\mathbf{n}}\right)$, that do commute with $H .^{25-27}$ We give one possible definition of such operators in Eq. (A1) in the Appendix. The operators $T_{A}\left(\mathbf{R}_{\mathbf{n}}\right)$, however, do not form a group and can thus not be directly used to construct the eigenstates of $H$. For a "rational field", where the number of magnetic flux quanta per unit cell, $\alpha=\frac{1}{2 \pi} \frac{e}{\hbar}(\mathbf{a} \times \mathbf{b}) B$, is a rational number, $\alpha=p / q$ with $p$ and $q$ relative prime, one can choose a larger magnetic lattice that has an integer number of $p$ flux quanta passing through each cell, e.g.,

$$
\mathbf{S}_{\mathbf{n}}=j \mathbf{a}+k(q \mathbf{b}), \quad \mathbf{n}=(j, k) \in \mathbb{Z}^{2} .
$$

On this lattice, a generalized version of the Bloch theorem holds,${ }^{27}$ for an orthorhombic lattice and Landau gauge, $\mathbf{A}(\mathbf{r})=$ $B x \mathbf{e}_{y}$, the solutions of Eq. (1) can be chosen of the form

$$
\psi(\mathbf{r}) \equiv e^{\mathrm{i} \theta \mathbf{r}} u^{\theta}(\mathbf{r})
$$

where $u^{\theta}(\mathbf{r})$ obeys

$$
u^{\theta}(\mathbf{r}+\mathbf{S})=\exp \left(-\mathrm{i} \frac{e}{\hbar} B S_{x} y\right) u^{\theta}(\mathbf{r}),
$$

and the magnetic crystal momentum $\boldsymbol{\theta}$ is restricted to the first magnetic Brillouin zone, i.e., the first Brillouin zone of Eq. (3). This condition allows to restrict the calculation of $u^{\theta}(\mathbf{r})$ to one primitive cell of the magnetic lattice. We have spelled out the steps leading to Eq. (5) in more detail in the Appendix.

\section{CALCULATION METHOD}

\section{A. Numerical solution of the Schrödinger equation}

Substituting $\psi(\mathbf{r})$ from Eq. (4) into Eq. (1) yields a differential equation for the unknown functions $u^{\theta}(\mathbf{r})$,

$$
H(\boldsymbol{\theta}) u_{j}^{\theta}(\mathbf{r}) \equiv[T(\boldsymbol{\theta})+V(\mathbf{r})] u_{j}^{\theta}(\mathbf{r})=E_{j}(\boldsymbol{\theta}) u_{j}^{\theta}(\mathbf{r}),
$$

where the kinetic energy operator $T(\boldsymbol{\theta})=\frac{1}{2 m}\left(\Pi_{x}^{2}+\Pi_{y}^{2}\right)$ in the Landau gauge chosen above has the form

$$
\Pi_{x}^{2}=-\hbar^{2}\left(\partial_{x}+\mathrm{i} \theta_{x}\right)^{2} ; \Pi_{y}^{2}=-\hbar^{2}\left(\partial_{y}+\mathrm{i} \theta_{y}+\frac{\mathrm{i} e}{\hbar} B x\right)^{2} .
$$

The symmetry property (5) can be exploited in a very elegant way: ${ }^{28}$ the periodicity of the functions $u^{\theta}(\mathbf{r})$ in the $y$-direction allows to expand them as a Fourier series,

$$
u^{\theta}(x, y)=\sum_{n=-\infty}^{\infty} \tilde{u}^{\theta}(x, n) \exp \left(\mathrm{i} 2 \pi \frac{n}{b q} y\right) .
$$

The Bloch condition (5) for the Fourier coefficients $\tilde{u}^{\theta}$ then reads

$$
\tilde{u}^{\theta}(x+a, n)=\tilde{u}^{\theta}(x, n+p),
$$

which reveals that every $p$ th coefficient function $\tilde{u}^{\theta}(x, n)$ is identical up to a shift of $a$ in the $x$-direction. For large $x$, the term proportional to $B^{2} x^{2}$ in $\Pi_{y}^{2}$ dominates over the periodic potential; the functions $\tilde{u}^{\theta}(x, n)$ thus decrease exponentially in this limit and only need to be considered on a finite interval.

To calculate the $n$ lowest eigensolutions of the eigenvalue problem (6), we have used the diffusion method: the evolution operator in imaginary time, $\mathcal{T}(\varepsilon)=\exp (-\varepsilon H)$, is repeatedly 
applied to a set of trial states $u_{j}(\mathbf{r}), j=1, \ldots, n$, which are orthogonalized after every step. To efficiently calculate the action of the operator exponential, we have used a recently developed high-order factorization scheme to split $\exp [-\varepsilon(T+V)]$ into terms that contain the exponentials of $T$ and $V$ alone. ${ }^{29}$ The exponential of $T=\frac{1}{2 m}\left(\Pi_{x}^{2}+\Pi_{y}^{2}\right)$ can be split further using an exact factorization scheme based on the harmonic-oscillator-like commutator relations of $\Pi_{x}^{2}$ and $\Pi_{y}^{2} \cdot{ }^{30}$ Using these two methods together, the resulting factorization of the evolution operator is

$$
\begin{aligned}
\mathcal{T}[- & \varepsilon H(\boldsymbol{\theta})] \\
= & \sum_{\ell=1}^{n} c_{\ell}\left[e^{-\frac{\varepsilon}{2 \ell} V} e^{-\frac{\varepsilon}{2 m \ell} C_{e}(\xi) \Pi_{y}^{2}} e^{-\frac{\varepsilon}{2 m \ell} C_{c}(\xi) \Pi_{x}^{2}}\right. \\
& \left.\times e^{-\frac{\varepsilon}{2 m \ell} C_{e}(\xi) \Pi_{y}^{2}} e^{-\frac{\varepsilon}{2 \ell} V}\right]^{\ell}+\mathcal{O}\left(\varepsilon^{2 n+1}\right),
\end{aligned}
$$

where the coefficients $c_{\ell}, C_{e}(\xi)$, and $C_{c}(\xi)$, with $\xi=\varepsilon \hbar e B / m$, are known analytically. ${ }^{29,30}$ We represent the wave functions $u_{j}^{\theta}(\mathbf{r})$ and the potential $V(\mathbf{r})$ by their values on an equidistantly spaced real-space grid. In Landau gauge, with the operators $\Pi_{x}^{2}$ and $\Pi_{y}^{2}$ defined as in Eq. (7), the product of operators in Eq. (10) is applied to a function $u^{\theta}(\mathbf{r})$ by performing the following steps (note that each step takes as input the result of the previous one):

(1) Calculate $e^{-\frac{\varepsilon}{2 \ell} V(\mathbf{r})} u^{\theta}(\mathbf{r})$; since the operator $V$ is diagonal in real space, this is simply a point-wise multiplication on the real-space grid.

(2) Calculate $\tilde{u}^{\theta}(x, n)$ by a fast Fourier transform (FFT) of $u^{\theta}(x, y)$ with respect to the $y$-component.

(3) In $(x, n)$ space, $e^{-\frac{\varepsilon}{2 m \ell} C_{e}(\xi) \Pi_{y}^{2}} \tilde{u}^{\theta}(x, n)$ is again a pointwise multiplication, see Eq. (7).

(4) Now regard $\tilde{u}^{\theta}(x, n)$ as a set of $p$ one-dimensional functions $g_{s}^{\theta}(x), s=1, \ldots, p$, in the sense of Eq. (9). Fouriertransforming these functions yields $p$ functions $\tilde{g}_{s}^{\theta}\left(k_{x}\right)$.

(5) Again, $e^{-\frac{\varepsilon}{2 m \ell} C_{c}(\xi) \Pi_{x}^{2}} \tilde{g}_{s}^{\theta}\left(k_{x}\right)$ is a simple point-wise multiplication.

(6) The remaining factors in the product can be applied by carrying out the above steps in reverse order.

Let us note here that the strategy we have followed to solve Eq. (6) is, of course, not unique, and that alternatives are possible. $^{31,32}$

\section{B. Quantized Hall conductance}

At a fixed rational field, $\alpha=p / q$, we obtain the bands $E_{j}(\boldsymbol{\theta})$ by numerically solving Eq. (6) for a grid of $\boldsymbol{\theta}$ values spanning the magnetic Brillouin zone (MBZ) corresponding to this field. The density of states (DOS) as a function of the field, $\rho(B, E)$, can then be calculated by integrating the bands over the MBZ and repeating the process for different fields. The rational field can only be tuned in discrete steps, $\alpha_{P}=P / Q$, where $P, Q \in \mathbb{Z}$. The size of the magnetic unit cell depends on the reduction of $P / Q$ to a quotient of relatively prime integers $p / q$, and is $q$ times as large as the zero-field ("geometric") unit cell. At the field $\alpha_{P}$, one zero-field band is thus expected to split into $q$ magnetic bands, which are known to cluster in groups of $p$ bands. ${ }^{2}$ The pattern of distinct prime factors of $P$ and $Q$ as $\alpha_{P}$ is swept across a range of fields gives rise to the self-similar, fractal pattern of gaps in the DOS that has become known as the Hofstadter butterfly.

When the Fermi energy of the system lies in a gap, i.e., a region where $\rho\left(B, E_{F}\right)$ is zero, the Hall conductance $\sigma_{x y}$ assumes a quantized value, $\sigma_{x y}^{\text {gap }}=n e^{2} / h, n \in \mathbb{Z}$. The fundamental topological reason for this quantization was revealed by Thouless et al. ${ }^{33}$ who showed that both in the strong field and strong potential limits the Kubo-Greenwood formula for $\sigma_{x y}$ is related to the Chern number of the U(1) bundle over the magnetic Brillouin zone. This was later $\operatorname{argued}^{34}$ to be a direct consequence of the magnetic translation symmetry, Eq. (5). Sweeping either the magnetic field or the Fermi energy through the fractal pattern of minigaps inside a broadened band or Landau level results in a peculiar, nonmonotonous Hall effect. ${ }^{33}$ Indications of this behavior have been found in experiments. ${ }^{3}$ In this work, we have used an alternative approach, introduced by $\mathrm{Strreda}^{35}$ to obtain $\sigma_{x y}^{\text {gap }}$ from the numerically calculated DOS,

$$
\sigma_{x y}^{\mathrm{gap}}\left(B, E_{F}\right)=\left.e \frac{\partial \rho\left(B, E^{\prime}\right)}{\partial B}\right|_{E^{\prime}=E_{F}} .
$$

\section{Low-field Hofstadter approximation}

In order to compare the results of the numerical solution of the Schrödinger equation to the well-established low-field approximation, we start with a TB model for the zero-field band structure of the square lattice,

$$
\begin{aligned}
E(\mathbf{k})= & \gamma_{n n}\left(e^{i k_{x} a}+e^{+i k_{y} a}+\text { c.c. }\right) \\
& +\gamma_{2 n n}\left(e^{i k_{x} a} e^{i k_{y} a}+e^{i k_{x} a} e^{-i k_{y} a}+\text { c.c. }\right) \\
& +\gamma_{3 n n}\left(e^{2 i k_{x} a}+e^{2 i k_{y} a}+\text { c.c. }\right)
\end{aligned}
$$

where "c.c." stands for the complex conjugate of the previous terms. Here, $\gamma_{n n}, \gamma_{2 n n}$, and $\gamma_{3 n n}$ are the first, second, and third nearest-neighbor hopping integrals, which are used as fitting parameters to reproduce a low zero-field band of the numerical calculation. Hopping integrals between more distant neighbors are neglected. One can then perform the Peierls substitution ${ }^{1,17} \mathbf{k} \rightarrow(\mathbf{p}+e \mathbf{A}) / \hbar$ in Eq. (12), where $\mathbf{p}$ is the momentum operator, using the Landau gauge $\mathbf{A}(\mathbf{r})=$ $B x \mathbf{e}_{y}$ for the vector potential. This transforms $E(\mathbf{k})$ into an effective Hamiltonian, which can be diagonalized by standard diagonalization methods, to obtain the magnetic dispersion relation, DOS and the Hall conductance in the mobility gaps $\sigma_{x y}^{\text {gap }}$ through Středa's formula.

\section{RESULTS AND DISCUSSION}

In our numerical calculations, we have studied a simple system consisting of a two-dimensional square lattice of potential wells with the symmetrized Fermi function form, ${ }^{36}$

$$
V(r)=U \operatorname{coth}\left(\frac{r_{0}}{2 d}\right) \frac{\sinh \left(\frac{r_{0}}{d}\right)}{\cosh \left(\frac{r}{d}\right)+\cosh \left(\frac{r_{0}}{d}\right)},
$$

with parameters

$$
r_{0}=39.7 \mathrm{~nm}, \quad d=1.59 \mathrm{~nm}, \quad a=100 \mathrm{~nm},
$$

where $a$ is the lattice spacing. The potential is illustrated in Fig. 1(a); the parameters are set to loosely reproduce the conditions of earlier experimental studies. 3,4 


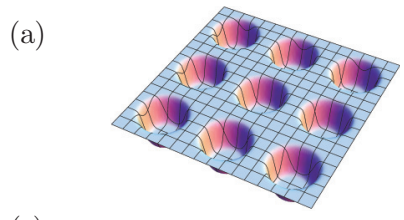

(c)

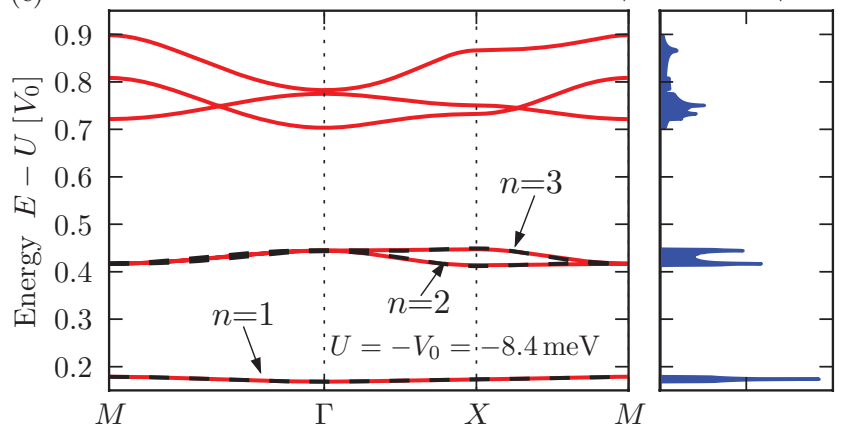

$|\mathbf{k}|$

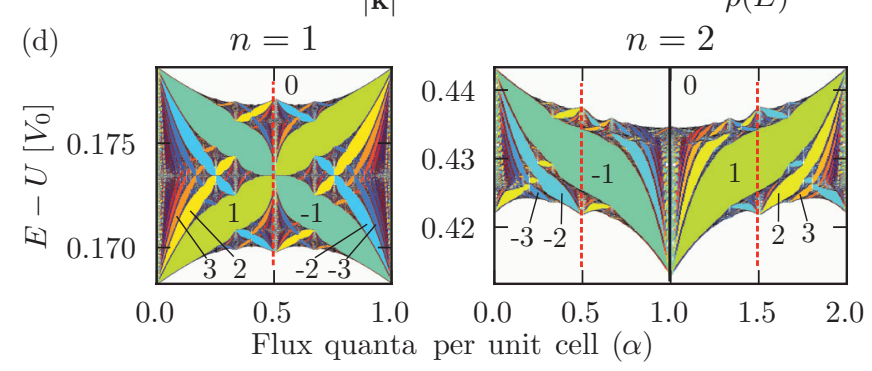

FIG. 1. (Color online) Zero-field band structure and TB approximation. (a) Schematic plot of the periodic Fermi well potential. (b) First Brillouin zone of the reciprocal lattice. (c) (Left) Zero-field band structure of the potential with parameters (14) and $U=-V_{0}$. Solid red lines show bands obtained by numerically solving the Schrödinger equation, dashed black lines are TB bands fitted to these exact bands (see text). (Right) DOS $\rho(E)$ obtained from the full Schrödinger equation. (d) Hofstadter butterflies for the two lowest bands obtained by Peierls substitution (see text). Areas with nonzero DOS are printed in black, and the gaps are colored according to the corresponding quantized Hall conductance $\sigma_{x y}^{\text {gap }}$ in units of $e^{2} / h$. White indicates zero Hall conductance, warm (cold) colors indicate positive (negative) Hall conductance. A number of larger gaps are labeled with the corresponding Hall conductance for reference. The butterflies are periodic in the flux, one period being shown in each case.

\section{A. Strong-potential regime}

We first compare the spectrum generated by the full Schrödinger equation (1) to the results of a TB approximation similar to the one used by Hofstadter. We thus choose a fairly deep modulation potential,

$$
U=-V_{0} \equiv-8.4 \mathrm{meV} \text {. }
$$

We obtained the band structure and DOS at zero magnetic field [see Fig. 1(c)] by numerically solving the corresponding single-particle Schrödinger equation. We then fitted the TB model Eq. (12) to each of the lowest three bands [black dashed lines in Fig. 1(c)], and employed the Peierls substitution to obtain the fractal energy spectra shown in Fig. 1(d). The fitting procedure was carried out over a regular grid of $\mathbf{k}$ points spanning the first Brillouin zone shown in Fig. 1(b). It was found that the lowest band [ $n=1$ in Fig. 1(c)] required only
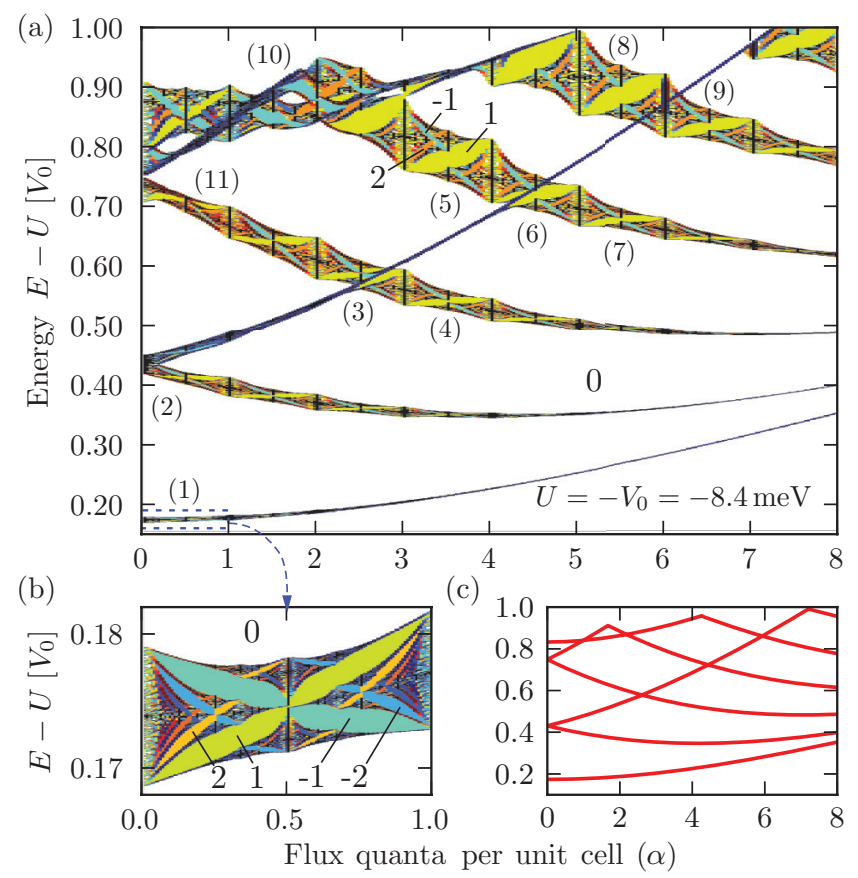

FIG. 2. (Color online) Full numerical calculation. Magnetic energy spectrum (DOS and Hall conductance $\sigma_{x y}^{\text {gap }}$ ) for the lowest six bands of the of the square Fermi well lattice with parameters (14) and $U=-V_{0}$, calculated by numerically solving Eq. (6). The color coding and labeling is as in Fig. (1). (b) Magnified portion of the lowest band indicated by the dashed box in (a). (c) Energy spectrum of an isolated Fermi well potential, Eq. (13). It strongly resembles the spectrum of a parabolic potential (the Fock-Darwin spectrum ${ }^{38}$ ).

nearest-neighbor hopping integrals in the TB band model for an adequate fit, and thus yields a spectrum corresponding to that obtained by Hofstadter ${ }^{2}$ [left panel of Fig. 1(d)]. The second and third bands required up to third-nearest-neighbor hoppings, which lead to significantly distorted versions of Hofstadter's butterfly, shown for the second band on the right panel of Fig. 1(d). The spectrum of the third band is similar to the second and is not shown. Our findings qualitatively agree with the results of Ref. 37.

In Fig. 2(a), we show the energy spectrum of the six lowest bands, as obtained by numerically solving the full magnetic eigenvalue problem, Eq. (6), using the scheme outlined in Sec. III A.

A maximum magnetic unit cell size of $Q=32$ was employed in the calculation, and $48 \times 48$ grid points for the functions $u_{j}^{\theta}(\mathbf{r})$ were used per geometric unit cell (i.e., $6 q$ states $u_{j}$ on a $q \times 48 \times 48$ grid had to be calculated at the field $\alpha_{P}=$ $P / Q=p / q$, where $p$ and $q$ are relative prime, see Sec. III B). For comparison, the spectrum of an isolated potential well is plotted in Fig. 2(c). It bears a strong resemblance to the Fock-Darwin (FD) spectrum ${ }^{38}$ of a parabolic well; we will thus refer to these states as "FD states" in the following. It can be seen in Fig. 2(a) that in the periodic system the FD states of the isolated well are broadened into bands with a fractal internal structure that is qualitatively well described by the Hofstadter butterfly. In the exact result, the periodicity of the Hofstadter spectrum is superimposed onto the field dependence of the corresponding FD state; this field-dependence is not taken into 

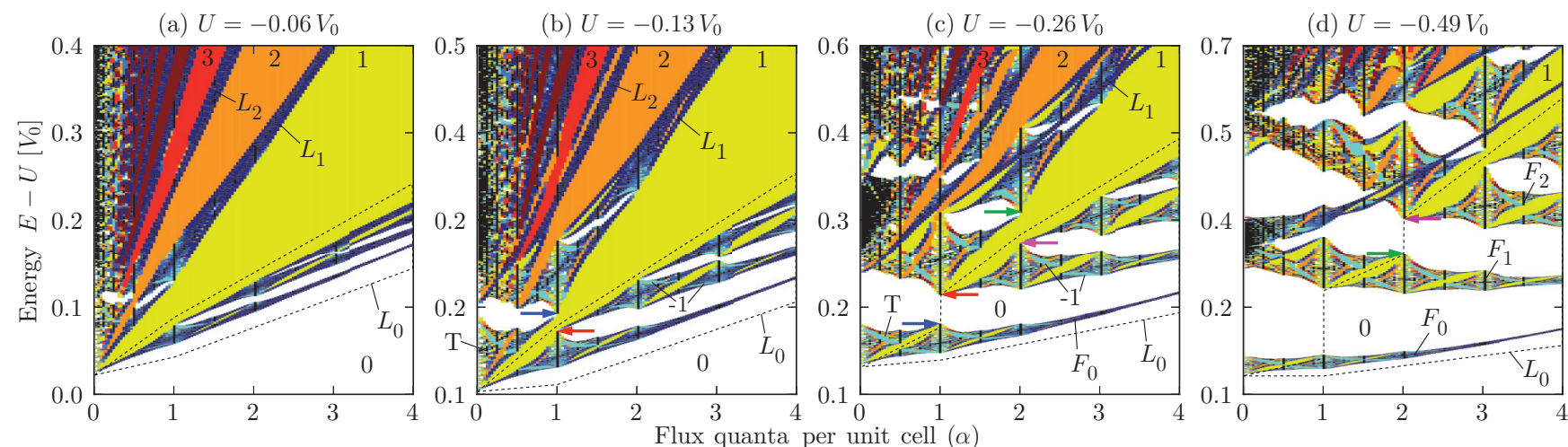

FIG. 3. (Color online) Energy spectra for different strengths of the modulation potential, ranging from a "Landau-like" system of nearly free electrons $\left[U=-0.06 V_{0}\right.$, (a) $]$ to a moderately deep potential $\left[U=-0.49 V_{0}\right.$, (d) $]$ already resulting in a "Fock-Darwin"-like spectrum. Energies and modulation potentials $U$ are given in units of $V_{0}$, the depth of the modulation potential used to generate Fig. 2. Gaps are color coded and labeled with their corresponding Hall conductance as in Fig. 1. Landau levels are denoted by $L_{1}, L_{2}, \ldots$, Fock-Darwin-like states $F_{0}, F_{1}, \ldots$ The dotted line encloses the broadened lowest Landau level. The label " $\mathrm{T}$ " indicates the triangular cluster of states referenced in the text.

account in the TB model with constant (i.e., field-independent) hopping integrals. In general, higher energy levels, having more extended wave functions, undergo larger broadening at a given flux value. Conversely, bands become narrower with increasing field, as their wave functions become more spatially localized, tending to Landau levels in the limit of high field intensities. In the TB model, the main effect of increasing the second and third nearest-neighbor hoppings is a distortion of the butterfly that opens a gap at flux strengths of $\alpha_{j}=j+1 / 2$, with $j \in \mathbb{Z}$, indicated by the vertical (red) dotted lines in the butterflies in Fig. 1(d). This behavior is also present in the exact results, compare regions (4)-(5) or (7)-(8) in Fig. 2(a). The gap widens for higher-energy bands, consistent with the TB approximation, where such bands need to be modeled by larger hopping integrals to more distant neighbors. The gap decreases again with increasing field due to the stronger localization of the wave functions, an effect that is not included in the TB description with $B$-independent hopping. Band crossings, which are not described within the single-band Peierls approximation, are an interesting subject for future studies: the narrow third FD band seems to disrupt the butterfly pattern of the broader bands it crosses in regions (3) and (6), but does not seem to exert any noticeable effect in region (9). At higher energies [see region (10)], where multiple bands cross, the resulting fractal spectrum can assume a form that is very different from the original Hofstadter butterfly.

\section{B. Intermediate-potential regime}

We now explore the evolution of the spectrum as the potential is changed from nearly flat (strong field limit) to highly modulated (strong potential limit). The intermediate stages of this evolution are not accessible to the approximate methodologies hitherto employed. We use the square lattice potential with the parameters given in Eq. (14), but change the well depth $U$. We start with a very shallow potential ( $U=-0.06 V_{0}$ ), which corresponds to a Landau-like system with nearly-free electrons in a magnetic field. The resulting spectrum is illustrated in Fig. 3(a); it exhibits the typical
"Landau fan" form, with slightly broadened Landau levels (LLs) that display an internal fractal structure of minigaps (most evident in the lowest level, $L_{0}$ ). The Hall conductance between LLs increases monotonically in steps of $e^{2} / h$, consistently with the integer quantum Hall effect. Furthermore, an emerging white gap $\left(\sigma_{x y}^{\text {gap }}=0\right)$ can be observed at low field $\left(\alpha=0-1, E \approx 0.11 V_{0}\right.$ ). When the potential modulation is increased to $U=-0.13 V_{0}$ [see Fig. 3(b)] this gap is seen to widen further, practically cutting off a low-energy triangular section (marked "T" in the figure) from all LLs $L_{n}$ with $n>0$. At $\alpha=1$, the upper-right tip of this triangular cluster of states retains a tenuous link to the broadened $L_{1}$ Landau band, at the position indicated by the blue arrow. At the same time, the minigaps at $\alpha \geqslant 1$ in the lowest Landau band $L_{0}$ have broadened further, to the point where the band is only held together by a narrow subband at $\alpha=1$, indicated by the red arrow. The Hall conductance in the gap between $L_{0}$ and $L_{1}$ at $\alpha=1$ is still $\sigma_{x y}^{\text {gap }}=1$. However, upon further increasing the modulation strength, the gap first closes and then reopens with the above links reversed: at $U=-0.26 V_{0}$ [see Fig. 3(c)], the tip of the triangular cluster is now connected to the lowest miniband of $L_{0}$ (blue arrow), while the rest of the $L_{0}$ miniband is connected to $L_{1}$ (red arrow). At the same time, the gap has changed its character to $\sigma_{x y}^{\text {gap }}=0$, resulting in the first clearly discernible FD-like band $F_{0}$, separated from all higher energy states by an unbroken white gap. Most of $F_{0}$ is formed from the lowest miniband of the LL $L_{0}$, except for the triangular cluster of states in $\alpha<1$, which originates from higher LLs. The process of formation of the first FD band $\left(F_{0}\right)$ that is incipient at $\alpha=1$ in Fig. 3(b) can be seen to repeat itself at $\alpha=2$ for the second FD band $\left(F_{1}\right)$ [Fig. 3(c), green and purple arrows] and at $\alpha=3$ for the third FD band $\left(F_{2}\right)$ [see Fig. $3(\mathrm{~d})$ ]. The gradual transformation from LLs to FD-like bands thus proceeds by the reconnection of minibands from one broadened LL to those of neighboring LLs. This rearrangement process fragments the large triangular gaps between LLs in the weak potential limit into minigaps encapsulated by the emerging FD-like bands. As a result, the FD states $F_{0}, F_{1}, F_{2}$ in Fig. 3(d) are composed of a low-field section that originates from higher LLs (triangle $\mathrm{T}$ in 
case of $F_{0}$, one Hofstadter butterfly segment plus a triangular cluster in case of $F_{1}$, etc.), and a high-field section that is one miniband of the lowest LL $L_{0}$.

\section{CONCLUSIONS}

The above results show that with the method presented here it is possible to perform exact calculations of the spectrum of independent electrons in a $2 \mathrm{D}$ periodic potential and constant perpendicular magnetic field. The same technique is readily applicable to solve the Kohn-Sham equations of density functional theory (DFT), which are expected to provide a reasonable description at least for weakly correlated electron systems. Experimental techniques have recently become available to directly probe the local DOS in 2DEGs on surfaces in a perpendicular magnetic field using scanning tunneling spectroscopy, making possible the detection of spatial features of some LLs, ${ }^{39}$ and even measuring their response to a $1 \mathrm{D}$ periodic potential due to surface buckling. ${ }^{40}$ Moiré superlattices ${ }^{5}$ constructed from layers of hexagonal BN and graphene have very recently been shown ${ }^{6,7}$ to hold promise as systems in which the spectral properties of the 2DEG subject to a periodic potential and a perpendicular magnetic field can be probed with experimentally accessible magnetic field intensities. Such developments may presently bring about additional possibilities of experimentally determining the spectral features of the 2DEG subject simultaneously to a periodic potential and a perpendicular magnetic field, contrasting them with the predictions reported herein. We anticipate that the computational procedure described here will be a valuable tool in the analysis and interpretation of such experiments.

\section{ACKNOWLEDGMENTS}

We wish to thank A. García and E. Krotscheck for helpful discussions. SJ was funded by the Austrian Science fund FWF under project no. J2936-N, E.R.H. by the Spanish Ministry of Science and Innovation through project FIS2009-12721C04-03. SJ would like to thank ICMAB and ICMM for their hospitality during his stay. We acknowledge CESGA and the Johannes Kepler University Linz for the use of their computer facilities, where the results reported here were obtained.

\section{APPENDIX: MAGNETIC TRANSLATION GROUP AND GENERALIZED BLOCH THEOREM}

To find symmetry operations that commute with the Hamiltonian $H$ in Eq. (1), we start by defining the conjugate momentum operator, $\overline{\mathbf{\Pi}}=\mathbf{p}+e \overline{\mathbf{A}}(\mathbf{r})$, where $\overline{\mathbf{A}}(\mathbf{r})$ is the conjugate vector potential. For linearly gauged vector potentials, i.e., $\mathbf{A}(\mathbf{r})=\mathcal{J} \mathbf{r}$ with a constant Jacobian $\mathcal{J}$, the conjugate potential is $\overline{\mathbf{A}}(\mathbf{r}) \equiv \mathcal{J}^{T} \mathbf{r}$. A more general definition can be found in the review article. ${ }^{27}$ With the conjugate momentum operator, we define a family of magnetic translation operators, ${ }^{25-27}$

$$
T_{A}\left(\mathbf{R}_{\mathbf{n}}\right) \equiv \exp (-\mathrm{i} \pi j k \alpha) \exp \left(\frac{\mathrm{i}}{\hbar} \overline{\boldsymbol{\Pi}} \cdot \mathbf{R}_{\mathbf{n}}\right),
$$

where $\alpha=\frac{1}{2 \pi} \frac{e}{\hbar}(\mathbf{a} \times \mathbf{b}) B$ is the magnetic flux through one unit cell in units of the flux quantum $\phi_{0}=h / e$, and $\mathbf{R}_{\mathbf{n}}$ is given by Eq. (2). These operators can be interpreted as translations with an additional gauge transform to reverse the effect of the translation on the vector potential. It is fairly straightforward to show that they commute with the Hamiltonian (1), and have the property

$$
T_{A}\left(\mathbf{R}_{2}\right) T_{A}\left(\mathbf{R}_{1}\right)=\exp \left(\mathrm{i} 2 \pi j_{1} k_{2} \alpha\right) T_{A}\left(\mathbf{R}_{1}+\mathbf{R}_{2}\right),
$$

where $\mathbf{R}_{1}=j_{1} \mathbf{a}+k_{1} \mathbf{b}$ and $\mathbf{R}_{2}=j_{2} \mathbf{a}+k_{2} \mathbf{b} .^{27}$ The operators $T_{A}\left(\mathbf{R}_{\mathbf{n}}\right)$ thus do not form a proper group, but a "group up to a phase factor". The situation is considerably simplified in the case of a rational field, $\alpha=p / q$, where $p$ and $q$ are relatively prime integers. In this case, the phase factor in Eq. (A2) is a $q$ th root of unity for any lattice translation, and the product of the cyclic group of $q$ th roots of unity and the set of operators $T_{A}\left(\mathbf{R}_{\mathbf{n}}\right)$ form a group, the so-called magnetic translation group $\mathcal{G}{ }^{27}$ The limit of irrational flux has also been studied by several authors, see, e.g., Ref. 41.

We now choose a subset of the lattice $\mathbf{R}_{n}$, Eq. (2), such that its (larger) primitive cell encloses a number $p$ of flux quanta, e.g.,

$$
\mathbf{S}_{\mathbf{n}}=j \mathbf{a}+k(q \mathbf{b}), \quad \mathbf{n}=(j, k) \in \mathbb{Z}^{2} .
$$

The phase factor in Eq. (A2) is then equal to one on this magnetic lattice, and the operators $T_{A}\left(\mathbf{S}_{\mathbf{n}}\right)$ form a normal Abelian subgroup of $\mathcal{G}$, from which its irreducible standard representations can be calculated. ${ }^{27}$ The zero-field Bloch situation is now almost restored: the unitary operators $T_{A}\left(\mathbf{S}_{\mathbf{n}}\right)$ fulfill $T_{A}\left(\mathbf{S}_{2}\right) T_{A}\left(\mathbf{S}_{1}\right)=T_{A}\left(\mathbf{S}_{1}+\mathbf{S}_{2}\right)$. Their eigenvalues thus must be of the form

$$
T_{A}\left(\mathbf{S}_{\mathbf{n}}\right) \phi(\mathbf{r})=e^{\mathrm{i} \theta \mathbf{S}_{\mathbf{n}}} \phi(\mathbf{r})
$$

with a constant vector $\boldsymbol{\theta}$, and we use the Bloch ansatz $\phi(\mathbf{r}) \equiv$ $e^{\mathrm{i} \theta \mathbf{r}} u^{\theta}(\mathbf{r})$ for their eigenfunctions. For the subsequent calculations, we use an orthorhombic lattice formed by vectors $\mathbf{a}=$ $a \mathbf{e}_{x}, \mathbf{b}=b \mathbf{e}_{y}$ and Landau gauge, $\mathbf{A}(\mathbf{r})=B x \mathbf{e}_{y}$. Substituting the definition of the magnetic translation operators, Eq. (A1), into Eq. (A4) yields the generalized Bloch condition ${ }^{27}$ for the functions $u^{\theta}(\mathbf{r})$,

$$
u^{\theta}(\mathbf{r}+\mathbf{S})=\exp \left(-\mathrm{i} \frac{e}{\hbar} B S_{x} y\right) u^{\theta}(\mathbf{r}),
$$

where the magnetic crystal momentum $\boldsymbol{\theta}$ can be restricted to the first magnetic Brillouin zone- $\pi / a \leqslant \theta_{x} \leqslant+\pi / a$, $-\pi /(q b) \leqslant \theta_{y} \leqslant+\pi /(q b)$. As $H$ commutes with the magnetic translations $T_{A}\left(\mathbf{S}_{\mathbf{n}}\right)$, we can seek its eigenstates among the family of functions $\phi(\mathbf{r})=e^{\mathrm{i} \theta \mathbf{r}} u^{\theta}(\mathbf{r})$, see Eq. (A4). The calculation of the eigenstates of $H$ is thus reduced to a finite domain, namely the first magnetic Brillouin zone. *stefan.janecek@ricam.oeaw.ac.at

${ }^{1}$ R. Peierls, Z. Physik 80, 763 (1933).

${ }^{2}$ D. R. Hofstadter, Phys. Rev. B 14, 2239 (1976).
${ }^{3}$ C. Albrecht, J. H. Smet, K. von Klitzing, D. Weiss, V. Umansky, and H. Schweizer, Phys. Rev. Lett. 86, 147 (2001). 
${ }^{4}$ M. C. Geisler, J. H. Smet, V. Umansky, K. von Klitzing, B. Naundorf, R. Ketzmerick, and H. Schweizer, Phys. Rev. Lett. 92, 256801 (2004).

${ }^{5}$ R. Bistritzer and A. H. MacDonald, Phys. Rev. B 84, 035440 (2011). ${ }^{6}$ L. A. Ponomarenko, R. V. Gorbachev, G. L. Yu, D. C. Elias, R. Jalil, A. A. Patel, A. Mishchenko, A. S. Mayorov, C. R. Woods, J. R. Wallbank, et al., Nature (London) 497, 594 (2013).

${ }^{7}$ C. R. Dean, L. Wang, P. Maher, C. Forsythe, F. Ghahari, Y. Gao, J. Katoch, M. Ishigami, P. Moon, M. Koshino, et al., Nature (London) 497, 598 (2013).

${ }^{8}$ U. Kuhl and H. J. Stockmann, Phys. Rev. Lett. 80, 3232 (1998).

${ }^{9}$ C. de Lange and T. Janssen, Phys. Rev. B 28, 195 (1983).

${ }^{10}$ M. Quilichini and T. Janssen, Rev. Mod. Phys. 69, 277 (1997).

${ }^{11}$ D. Jaksch and P. Zoller, New J. Phys. 5, 56 (2003).

${ }^{12}$ K. Fang, Z. Yu, and S. Fan, Nat. Photon. 6, 782 (2012).

${ }^{13}$ M. Hafezi, E. A. Demler, M. D. Lukin, and J. M. Taylor, Nat. Phys. 7, 907 (2011).

${ }^{14}$ P. G. Harper, P. Phys. Soc. Lond. A 68, 874 (1955).

${ }^{15}$ D. Langbein, Phys. Rev. 180, 633 (1969).

${ }^{16}$ G. H. Wannier, G. M. Obermair, and R. Ray, Phys. Status Solidi B 93, 337 (1979).

${ }^{17}$ J. M. Luttinger, Phys. Rev. 84, 814 (1951).

${ }^{18}$ Y. Hasegawa, P. Lederer, T. M. Rice, and P. B. Wiegmann, Phys. Rev. Lett. 63, 907 (1989).

${ }^{19}$ V. N. Nicopoulos and S. A. Trugman, Phys. Rev. Lett. 64, 237 (1990).

${ }^{20}$ A. S. Alexandrov and H. Capellmann, Phys. Rev. Lett. 66, 365 (1991)

${ }^{21}$ G. A. Vugalter and A. S. Pastukhov, J. Phys. A: Math. Gen. 37, 5763 (2004).

${ }^{22}$ W. Kohn, Phys. Rev. 115, 1460 (1959).
${ }^{23}$ D. Springsguth, R. Ketzmerick, and T. Geisel, Phys. Rev. B 56, 2036 (1997).

${ }^{24}$ O. Kühn, V. Fessatidis, H. L. Cui, P. E. Selbmann, and N. J. M. Horing, Phys. Rev. B 47, 13019 (1993).

${ }^{25}$ E. Brown, Phys. Rev. A: Gen. Phys. 133, 1038 (1964).

${ }^{26}$ J. Zak, Phys. Rev. A: Gen. Phys. 134, 1607 (1964).

${ }^{27}$ H. J. Fischbeck, Phys. Status Solidi 38, 11 (1970).

${ }^{28}$ W. Cai and G. A. Galli, Phys. Rev. Lett. 92, 186402 (2004).

${ }^{29}$ S. A. Chin, S. Janecek, and E. Krotscheck, Chem. Phys. Lett. 470, 342 (2009).

${ }^{30}$ M. Aichinger, S. A. Chin, and E. Krotscheck, Comput. Phys. Commun. 171, 197 (2005).

${ }^{31}$ A. Trellakis, Phys. Rev. Lett. 91, 056405 (2003).

${ }^{32}$ J. Bellissard, H. van Elst, and H. S. Baldes, J. Math. Phys. 35, 5373 (1994).

${ }^{33}$ D. J. Thouless, M. Kohmoto, M. P. Nightingale, and M. den Nijs, Phys. Rev. Lett. 49, 405 (1982).

${ }^{34}$ I. Dana, J. E. Avron, and J. Zak, J. Phys. C Solid State 18, L679 (2000).

${ }^{35}$ P. Streda, J. Phys. C Solid State 15, L717 (1982).

${ }^{36}$ D. W. L. Sprung and J. Martorell, J. Phys. A: Math Gen 30, 6525 (1997).

${ }^{37}$ F. Claro, Phys. Status Solidi B 104, K31 (1981).

${ }^{38}$ C. G. Darwin, Proc. Cambridge Philos. Soc. 27, 86 (1930).

${ }^{39}$ K. Hashimoto, T. Champel, S. Florens, C. Sohrmann, J. Wiebe, Y. Hirayama, R. A. Römer, R. Wiesendanger, and M. Morgenstern, Phys. Rev. Lett. 109, 116805 (2012).

${ }^{40}$ Y. Okada, W. Zhou, C. Dhital, D. Walkup, Y. Ran, Z. Wang, S. D. Wilson, and V. Madhavan, Phys. Rev. Lett. 109, 166407 (2012).

${ }^{41}$ G. M. Obermair and G. H. Wannier, Phys. Status Solidi B 76, 217 (1976). 